\title{
Epidemio-clinical, etiological, therapeutic and evolutive profile of hyperhidrosis: Moroccan experience
}

\section{Mohamed El Amraoui', Abdelhafid Achbouk², Rachid Frikh'1, Naoufal Hjira1, Mohammed Boui'}

${ }^{1}$ Dermatology-Venereology, Mohammed V Military Teaching Hospital, Rabat, Morocco, ${ }^{2}$ Plastic Surgery, Mohammed V Military Teaching Hospital, Rabat, Morocco

Corresponding author: Dr. Mohamed El Amraoui, E-mail: med.elamraoui.dto@gmail.com

Sir,

Hyperhidrosis (HH) is an excessive production of sweat which is responsible for aesthetic and social discomfort with a major impact on the quality of life and an increased frequency of infectious complications $[1,2]$. Despite its relative rarity, it is sometimes a diagnostic and therapeutic puzzle for the dermatologist. We present this prospective descriptive study, conducted over a period of 12 months (June 2017-May 2018), within the dermatology-venereology department of the Mohammed V military training hospital, according to a pre-established operating file.

21 cases were reported, including $65 \%$ of women and $35 \%$ of men, age of patients ranged between 13 and 44 years with an average of 27.41 years, $59 \%$ were of average social class and $41 \%$ of class social low. $94 \%$ of $\mathrm{HH}$ were localized, $6 \%$ were generalized and no case of regional $\mathrm{HH}$. The most frequent locations were: $65 \%$ palmar, 35\% axillary and 29\% plantar (Fig. 1). 80\% of patients had HH that started with puberty, $41 \%$ had an anxiety state and 35\% had family cases. The severity according to the HDSS score was: level I $0 \%$, level II $35 \%$, level III $41 \%$ and level IV 23\%. The treatments used were antiperspirants $65 \%$, Ionophoresis $29 \%$, Oxybutynin 35\%, Botulinum toxin $41 \%$ and thoracic sympathectomy $23 \%$. The effectiveness of treatments ranged from none to $100 \%$ with an average of $64 \%$, the most effective treatments were botulinum toxin and thoracic sympathectomy, the least effective treatments were antiperspirants and Oxybutynin,

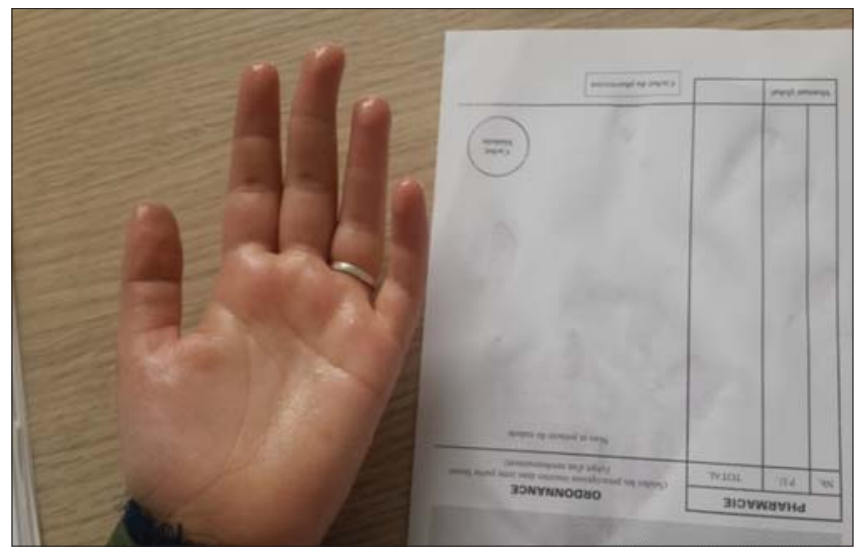

Figure 1: Palmar hyperhidrosis in a 16-year-old girl.

while ionophoresis is in the intermediate zone. Patient satisfaction ranged from $3 / 10$ to $10 / 10$ with an average of 7.17/10. Tolerance of treatments ranged from $7 / 10$ to $10 / 10$ with an average of $8.7 / 10$. The side effects found were compensatory body sweating following thoracic sympathectomy in one patient.

HH in our context is characterized by: young age of onset, female predominance, prevalence of localized forms, frequency of familial forms, frequency of moderate to severe forms, ineffectiveness of antiperspirants and Oxybutynin, the efficacy of botulinum toxin and sympathectomy, a very good satisfaction and tolerance and the scarcity of side effects. HH profoundly impacts the psychic state of particularly young and adolescent patients [1-5]. The mild forms can be managed by antiperspirants, Oxybutynin and iontophoresis while

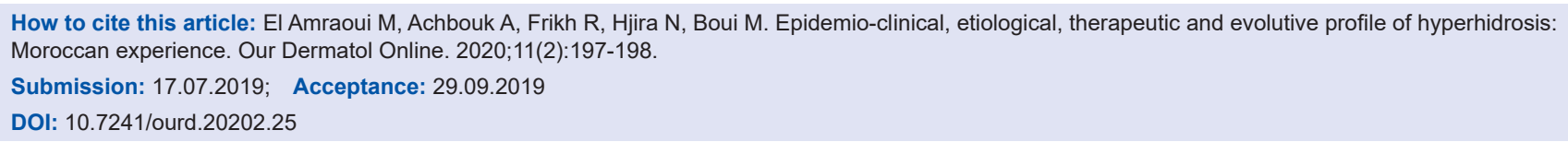


moderate and severe forms justify the use of botulinum toxin or even thoracic sympathectomy and hence the value of multidisciplinary collaboration.

\section{Consent}

The examination of the patient wasconductedaccording to the Declaration of Helsinki principles.

\section{REFERENCES}

1. Maillard H, Lecouflet M. Management of hyperhidrosis. Ann Dermatol Vénéréol. 2015;142:252-61.

2. Kouris A, Armyra K, Christodoulou C, Karimali P,
Karypidis D, Kontochristopoulos G. Quality of life in patients with focal hyperhidrosis before and after treatment with botulinum toxin A. ISRN Dermatol. 2014;2014:308650.

3. Menzinger S, QuenanS. Evaluation et prise en charge de l'hyperhidrose. Rev Med Suisse. 2017;13:710-4.

4. Mordant P, Castier Y, Maury JM, Karsenti A, Paraskevas N, Cerceau P, et al. Prise en charge de l'hyperhidrose primitive invalidante. Sang Thrombose Vaisseaux. 2010;22:302-10.

5. Kettle C, Freiberg A. Axillary hyperhidrosis treatment by simple skin excision and undermining. Can J Plast Surg. 1999;7:267-72.

Copyright by Mohamed El Amraoui, et al. This is an open-access article distributed under the terms of the Creative Commons Attribution License, which permits unrestricted use, distribution, and reproduction in any medium, provided the original author and source are credited.

Source of Support: Nil, Conflict of Interest: None declared. 